\title{
Using Microscopy to Qualitatively and Quantitatively Assess Crystalline Content in Amorphous Active Pharmaceutical Ingredients and Drug Product
}

\author{
Andrew D. Vogt and Joseph P. Neilly
}

AbbVie Inc, Drug Product Development, NCE Analytical R\&D, North Chicago, IL USA.

Amorphous active pharmaceutical ingredients (APIs) have improved solubility characteristics over their crystalline analogs. As consequence, amorphous APIs can have improved bioavailability. These improved properties often come at the expense of chemical and physical stability for the API [1]. The presence and quantity of the crystalline form in the amorphous material should be assessed to ensure manufacturing reproducibility and high quality API for its intended use. It is also important to monitor crystalline API in an amorphous drug product to ensure there is no form conversion that might affect the drug product (DP) over its shelf life.

Amorphous materials have no long-range order compared to their crystalline analogs and as a result can be identified by the absence of any sharp peaks in an x-ray powder diffraction (XRPD) pattern (Figure 1). The presence of sharp peaks in a diffraction pattern indicates that a material has crystalline character. XRPD has been able to detect crystalline content in amorphous API down to about 2\% w/w [2], but this is highly dependent on the API. In addition to XRPD, other techniques that have been used to identify and/or quantify crystalline content in amorphous API include the following [2-5]:

- Differential scanning calorimetry

- Fourier transform infrared spectroscopy

- $\quad$ Near infrared spectroscopy
- Raman spectroscopy

- Solid State Nuclear Magnetic Resonance

- Polarized Light Microscopy

Each of these techniques has advantages and disadvantages on their ability to differentiate between crystalline and amorphous phases of an API. Important considerations when characterizing DP with an amorphous API for crystalline content is drug load and formulation matrix. Since there are multiple components in a DP (API and excipients), specificity is very important for identifying a crystalline API and differentiating it from all other components. Thus, the spectroscopic techniques have advantage for specificity, but their sensitivities for a specific API will vary.

Polarized light microscopy (PLM) is very good at determining the presence of very small quantities of crystalline material in an amorphous API [6]. However, the technique cannot easily discern crystalline material that is API from other crystalline materials that may be present in the formulation. Hot stage microscopy using PLM can be used to help to overcome this limitation if the melting point of the crystalline material and the melting point of materials in a formulation matrix are known. Figure 2 shows that the crystalline material in this solid dispersion melted before the melting point of the API and, therefore, is not crystalline API.

Other imaging/mapping-based techniques that have shown promise in discerning crystalline API from amorphous API are elemental mapping by energy dispersive x-ray spectroscopy and chemical imaging by Raman spectroscopy [7]. Relatively newer technologies including micro-CT and second order near field imaging of chiral crystals (SONICC) are being evaluated for identification and quantification of 
crystalline form in an amorphous API. The use of these imaging based techniques for distinguishing crystalline API from amorphous API will be discussed.

\section{References:}

[1] J. Bauer, J. Validation Tech. (2009), pp. 63-68.

[2] B.A. Sarsfield et al. JCPDS-International Centre for Diffraction Data 2006 ISSN 1097-0002.

[3] S Hogan, G Buckton Pharm Res. 18(1) (2001), pp. 112-116.

[4] Solid-State NMR Application Note: Durham University, Solid-State NMR Research Service, 2007.

[5] U Zimper et al., Pharmaceutics 2 (2010), pp. 30-49.

[6] J Bauer et al., Pharm Res. 18(6) (2001), pp. 859-866.

[7] J. P. Neilly, et al., Microsc. Microanal. 17 (Suppl. 2) (2011) 1130-1131.
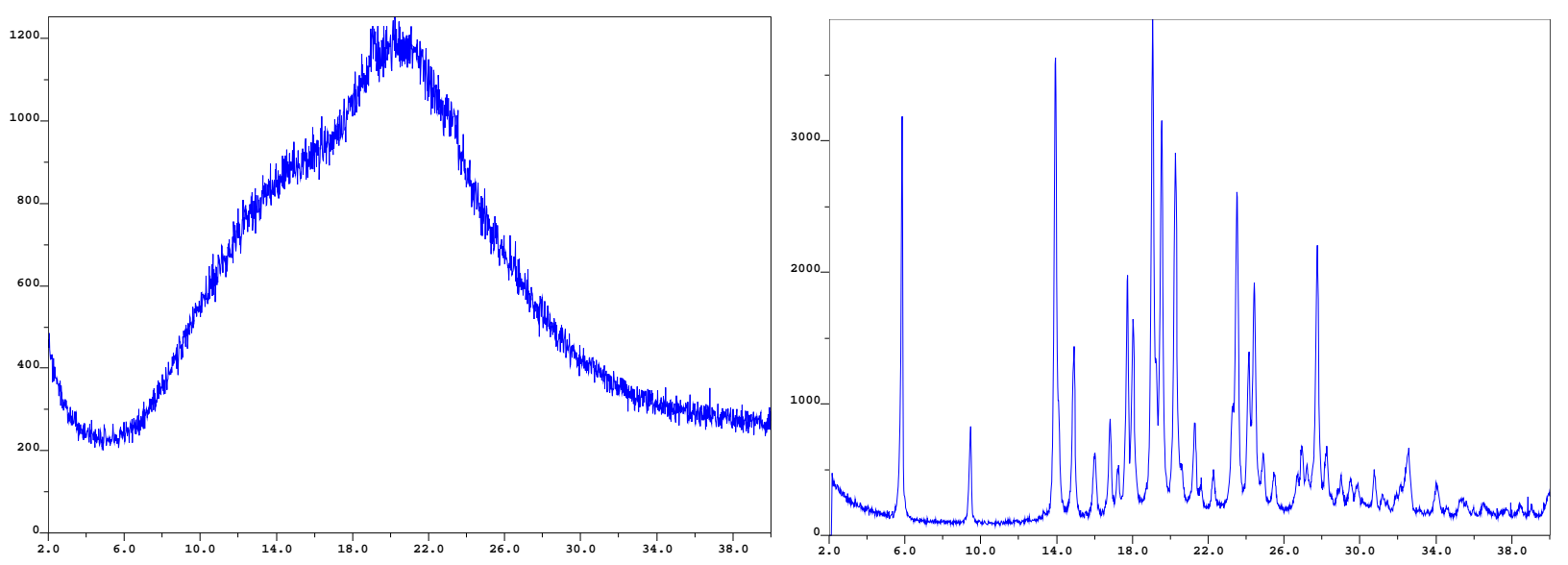

Figure 1. X-ray patterns of amorphous (left) and crystalline (right) form of the same API.
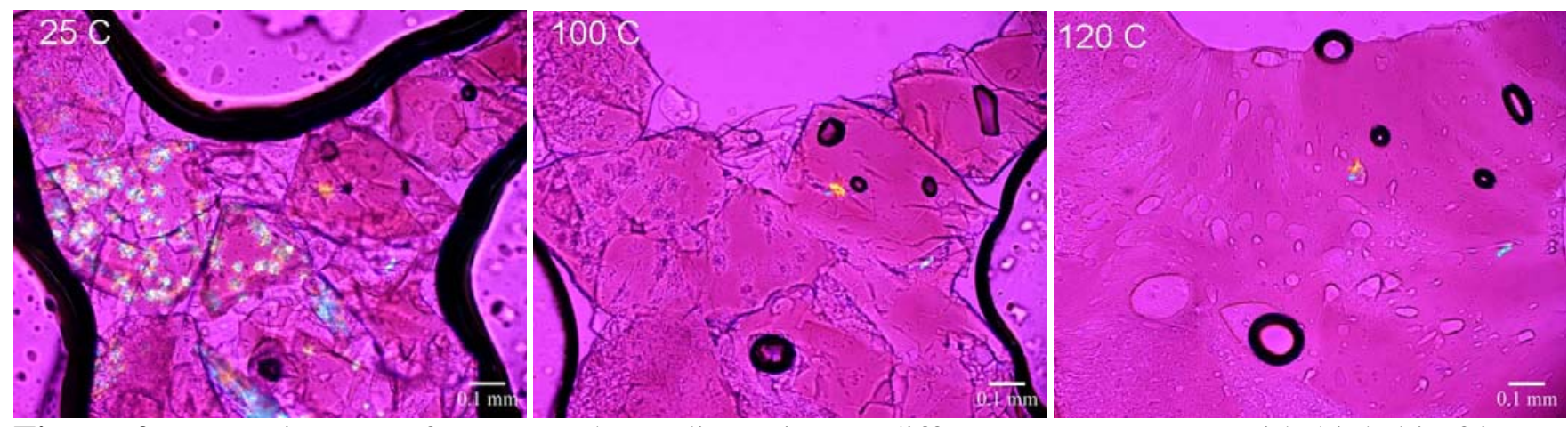

Figure 2. PLM images of an amorphous dispersion at different temperatures with high birefringent features, suggesting crystalline material. Since the melting point of the material is $\sim 125{ }^{\circ} \mathrm{C}$, the crystalline material is not API. XRPD of the same material indicated the material to be amorphous.

\section{Disclosures:}

Authors are employees of AbbVie and may own AbbVie stock. The design, study conduct, and financial support for this research were provided by AbbVie. AbbVie participated in the interpretation of data, review, and approval of the publication. 